\section{Correção endoscópica de fístula liquórica rinogênica: experiência de 44 casos}

\author{
Bernardo Cunba Araujo Filbo', Ossamu Butugan', \\ Francini Grecco de Melo Pádua ${ }^{3}$, \\ Richard Louis Voegels ${ }^{4}$
}

\section{Resumo / Summary}

\begin{abstract}
$\mathbf{A}$
comunicação entre o espaço subaracnóideo e a cavidade nasal ou seios frontal, etmoidal e esfenoidal denomina-se fístula liquórica rinogênica. A fístula liquórica rinogênica tem potencial de complicação com importante morbidade e mortalidade. Ela pode apresentar um verdadeiro desafio no diagnóstico, na sua localização e no seu tratamento. Forma de estudo: estudo de série. Material e método: Entre 1993 a 2004, 44 pacientes com fístula liquórica rinogênica foram submetidos à abordagem endoscópica no Hospital das Clínicas da Universidade de São Paulo. Os prontuários de todos os pacientes tratados foram revisados. Resultado: Quarenta e quatro pacientes, 16 mulheres (36\%) e 28 homens (64\%) foram incluídos no estudo. A idade variou de 02 a 68 anos (média $=40,3$ ). A etiologia, o sítio da fístula, o diagnóstico, a técnica empregada e o seguimento pós-operatório foram discutidos. Conclusão: Os autores concluíram que a técnica cirúrgica endoscópica endonasal para o tratamento de fístula liquórica rinogênica apresenta elevado sucesso, baixa morbidade e resultados em longo prazo confiáveis.
\end{abstract}

\section{Endoscopic repair of CSF rhinorrhea: experience of 44 cases}

Palavras-chave: fístula liquórica, cirurgia endoscópica, endoscópio.

Key words: cerebrospinal fluid leakage, endoscopic surgery, endoscope. erebrospinal fluid (CSF) rhinorrhea is a leakage of fluid from the subarachnoid space to the frontal, sphenoidal or ethmoidal sinuses. CSF rhinorrhea is a known potential complication with significant morbidity and mortality. It may present a significant challenge in diagnosis, localization and management. Study design: series study. Material and method: Between 1993 and 2004, 44 patients with cerebrospinal rhinorrhea were operated on using intranasal endoscopic approach in the University Hospital of the University of Sao Paulo, Medical School. The charts of all patients treated in our hospital were reviewed. Results: Forty-four patients, 16 women (36\%) and twenty-eight men (64\%), were included in the study. Patients' ages ranged from 2 to 68 years (mean: 40.3 years). Etiology, site of leakage, diagnosis, technique, cause of failure and follow-up are discussed. Conclusion: The authors concluded that transnasal endoscopic surgery for CSF rhinorrhea had high success rate, low morbidity and stable long-term results.

\footnotetext{
${ }^{1}$ Otorrinolaringologista (residência no HCFMUSP), Especialista em ORL pela SBORL pós-graduando (doutorado) da Divisão de Clínica Otorrinolaringológica do HCFMUSP. ${ }^{2}$ Professor Associado da Disciplina de Otorrinolaringologia da Faculdade de Medicina da Universidade de São Paulo.

${ }^{3}$ Fellow em cirurgia endoscópica pela disciplina de Otorrinolaringologia da FMUSP; Fellow em cirurgia endoscópica pela Disciplina de Otorrinolaringologia da Universidade de Graz, Áustria; Pós-graduanda da Disciplina de Otorrinolaringologia da FMUSP.

${ }^{4}$ Professor Associado da Disciplina de Otorrinolaringologia da Faculdade de Medicina da Universidade de São Paulo. Artigo recebido em 08 de março de 2005. Artigo aceito em 08 de junho de 2005.
} 


\section{INTRODUCÃ̃}

A fístula liquórica (FL) foi descrita pela primeira vez por Galen, $200 \mathrm{AC}^{1,2}$. Saintclair Thompson relatou a primeira série de pacientes com fístula espontânea em 1889¹. Várias tentativas de corrigir a FL foram realizadas no século XX, porém é atribuído a Dandy, em 1926, o primeiro sucesso cirúrgico na correção de uma fístula, no qual ele suturou a fáscia lata sobre o defeito dural, por trás da parede posterior do seio frontal, via intracraniana ${ }^{2}$. Em 1964, Vrabec and Hallberg descreveram o uso da cirurgia endonasal na correção cirúrgica de uma FL na lâmina cribriforme ${ }^{2,3}$.

A fístula liquórica pode apresentar diversos sintomas e/ou sinais, entretanto o mais freqüente é a rinorréia ${ }^{4}$. Além disso, a presença da FL representa um risco de vida ao paciente, podendo ser ponto de partida para infecções no sistema nervoso central, como as meningites ${ }^{2,4}$. Desta forma, sua correção deve ser realizada nos casos em que não ocorra uma resolução espontânea. Desde Wigand, em 1981, que utilizou o endoscópio para reparar as fístulas, seu uso tem sido amplamente difundido ${ }^{4}$.

Atualmente, com os avanços na cirurgia de base de crânio e a introdução da cirurgia endoscópica funcional dos seios paranasais na prática cirúrgica do otorrinolaringologista, têm proporcionado um aumento na incidência de complicações severas, como por exemplo, a fístula liquórica5. Neste contexto, a duroplastia endonasal tem sido usada na correção de um grande número de lesões na dura-máter com grande sucesso e baixo índice de morbidade ao paciente $2,6,7$.

Neste artigo apresentamos nossa experiência, de 44 casos, na correção de fístula liquórica com o uso do endoscópio.

\section{CASUÍSTICA E MÉTODO}

Entre 1993 e 2004, 44 pacientes, com diagnóstico de fístula liquórica rinogênica foram submetidos à cirurgia endoscópica endonasal no Hospital das Clinicas da Faculdade de Medicina da Universidade de São Paulo. Os prontuários de todos os pacientes tratados em nosso serviço foram revisados. Quarenta e quatro pacientes, 16 mulheres (36\%) e 28 homens (64\%) foram incluídos no estudo. A idade variou de 02 a 68 anos (média: 40,3). Informações a respeito da causa, sítio da lesão dural, técnica de reparo, complicações e seguimento foram colhidas dos prontuários (Tabela 1).

Em todos os casos, a flurosceína foi aplicada por punção lombar, pouco antes da cirurgia, para facilitar a identificação da lesão dural e verificar o sucesso da correção durante o procedimento (Figura 1). Foi aplicada uma solução de flurosceína a 5\%, estéril, na região intratecal por punção lombar. Nós utilizamos $0,1 \mathrm{ml}$ de solução/ 10kg de massa corporal (não excedendo $1 \mathrm{ml}$ ) diluída em água destilada até
$10 \mathrm{ml}$. As técnicas "onlay" e "underlay" foram os procedimentos utilizados na correção do defeito dural. Todas as fístulas foram reparadas com tecidos conectivos, como os flaps de mucosa livre (Figura 2), flaps pediculados, dura liofilizada, pericárdio bovino ou fáscia lata e foram fixados

Tabela 1. Causa de sítio de fístula liquórica nos pacientes tratados com a técnica endonasal $(n=44)$

\begin{tabular}{lc}
\hline \multicolumn{2}{c}{ Sítio da fístula Etmóide } \\
Causa da FLN+Cribriforme & Esfenóide Frontal \\
\hline Pós-operatório & 66 \\
Trauma & 862 \\
Fístula congênita (meningoencefalocele) & 1367 \\
Espontânea & 945 \\
Pós exérese de Adenoma de hipófise & 88 \\
\hline TOTAL & 4422202 \\
\hline
\end{tabular}

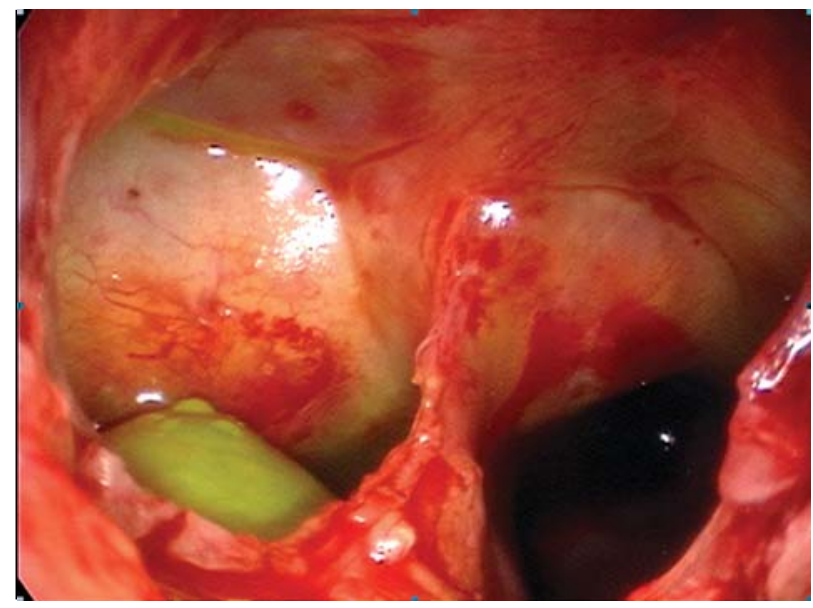

Figura 1. Fístula em seio esfenóide direito, drenando líquor marcado com flurosceína (verde).

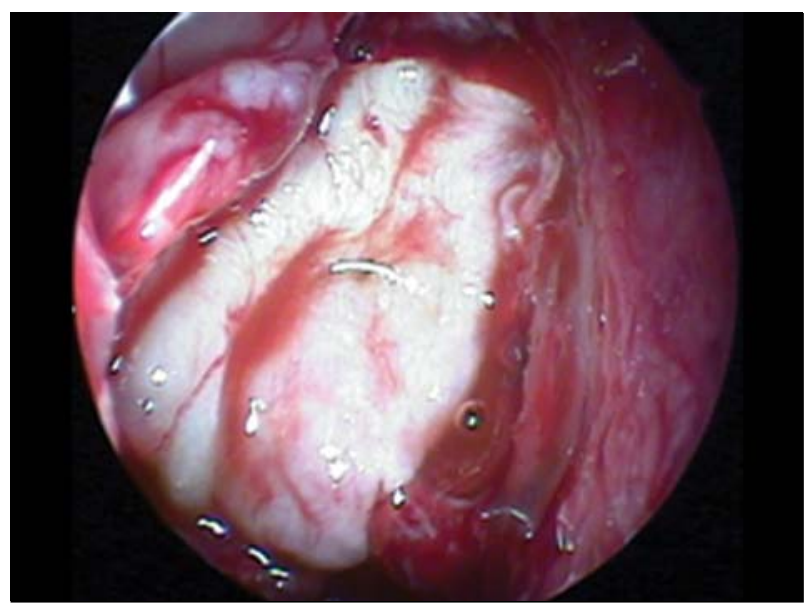

Figura 2. Flap livre de muco-pericôndrio utilizado em técnica "onlay". 
utilizando cola de fibrina e Surgicel®. Para a verificação do sucesso ou insucesso do procedimento, exames clínicos, controles endoscópicos e quando necessária endoscopia nasal, com uso de flurosceína, foi realizada no pós-operatório tardio.

\section{RESULTADOS}

As causas de fístula liquórica e os sítios em nossa série estão listados na Tabela 1. O sítio mais freqüente de fístulas foi a área etmóide-cribriforme. A meningoencefalocele foi a causa mais prevalente de fístula.

A técnica "underlay" foi utilizada em 4 casos (9\%), enquanto a técnica "onlay" foi realizada em 40 casos (91\%) (Figura 3). Nos casos em que utilizamos a técnica "underlay" também foi utilizada a técnica "onlay". Quando a FL era localizada nos seio esfenóide, este era preenchido com Surgicel ${ }^{\circledR}$.

Utilizamos dura liofilizada em 27 casos e usamos flap mucoso em 39 casos. Fáscia lata foi utilizada em 2 casos. Foi realizada derivação lombar externa (DLE) em 35 pacientes.

Os pacientes permaneciam em permaneceram em repouso relativo e decúbito com inclinação máxima de 10 graus por 5 dias em média e a DLE foi mantida em média por 4 dias. Dieta laxativa e orientações contra Vasalva eram oferecidas aos pacientes. Não utilizamos tampões nasais e antibiótico profilático foi introduzido no momento da indução anestésica. Optamos pela utilização de ceftriaxona, cefalosporina de terceira geração com penetração na barreira hemato-encefálica.

O seguimento pós-operatório variou de 2 a 102 meses (média: 39,4 meses).

A primeira correção cirúrgica da FL falhou em 9 casos. Entre estes pacientes, sete foram submetidos à nova abordagem endoscópica, enquanto 2 casos, que apresentavam falha óssea ampla foram submetidos a craniotomia para correção da fístula, pelo serviço de neurocirurgia.

Apenas um paciente desenvolveu meningite no pósoperatório, porém melhorou completamente após tratamento com antibióticos. Um dos pacientes desenvolveu síndrome de hiper-drenagem de líquor, ocasionada pelo desnível da bolsa coletora em relação ao tronco corporal. Todos os outros pacientes evoluíram bem sem complicações maiores.

\section{DISCUSSÃO}

A comunicação entre o espaço subaracnóideo e a cavidade nasal denomina-se fístula liquórica rinogênica ${ }^{1,8}$. Esta comunicação pode ocorrer diretamente entre a fossa craniana anterior e a cavidade nasal, ou indiretamente da fossa média e posterior, através da tuba auditiva ${ }^{1}$. As FL podem ser classificadas em traumáticas e não-traumáticas ${ }^{1,9}$. Em nossa série, 14 (32\%) das FL foram ocasionadas por trauma (6 traumas cirúrgicos e 8 traumas não-cirúrgicos), e

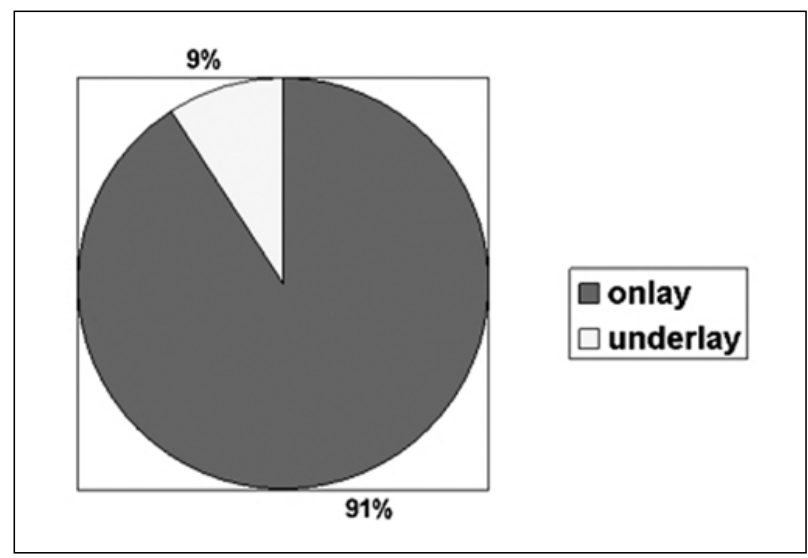

Figura 3. Técnica empregada no reparo da fístula.

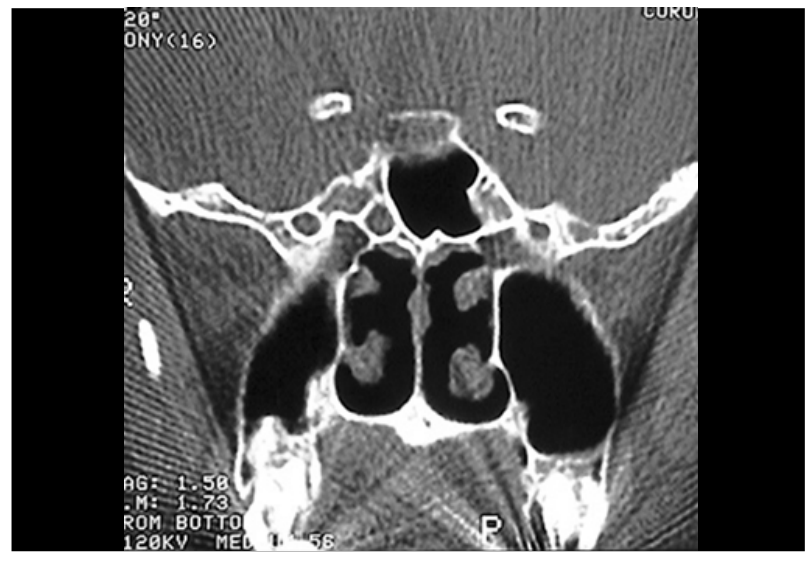

Figura 4. Tomografia de paciente com fístula liquórica em meningocele de seio esfenóide.

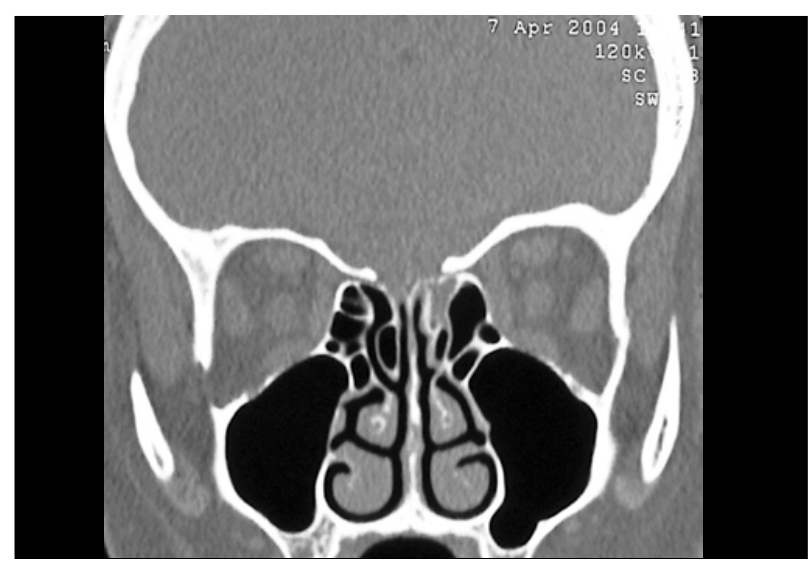

Figura 5. Tomografia de paciente com fístula liquórica em lâmina cribriforme durante sinusectomia etmoidal $\mathrm{E}$. 
o sítio mais acometido foi a lâmina cribriforme e a região do etmóide posterior, onde há um osso mais fino e onde a adesão da dura a este osso é maior ${ }^{1}$ (Figura 4). A presença de FL espontânea varia bastante na literatura, de 4 a 39\% ${ }^{6}$. Nossos achados foram semelhantes aos de Wax et al. (1997), onde as fístulas espontâneas representaram 29,5\% de nossa população. A origem das FL espontâneas não está bem esclarecida, existem alguns estudos que tentam explicar: como ser apenas um defeito congênito; representar uma pequena meningocele que é erodida pelo osso ou até mesmo ser derivada da atrofia focal de filamentos do nervo olfatório na lâmina cribriforme ${ }^{1,6}$. Durante a avaliação préoperatória, todos os pacientes foram submetidos à tomografia computadorizada, com cortes axiais e coronais, dos seios paranasais, para localização do sítio de lesão. Não utilizamos a B2-transferrina, que é o teste mais sensível para confirmação da $\mathrm{FL}^{5}$, por não haver disponibilidade, e a ressonância magnética (RM) foi solicitada apenas nos casos suspeitos de meningoencefaloceles, diferentemente ao que foi proposto nos estudos de Nachtigal e Schick, que preconizam RM em todos os $\operatorname{casos}^{2,4}$. A tomocisternografia também não foi utilizada nos casos inconclusivos, optando-se pela realização de infusão de flurosceína intratecal e diagnóstico sob visualização endoscópica. Todos os pacientes apresentavam história de rinorréia com líquido tipo "água de rocha" por uma narina e a confirmação, sob visualização endoscópica, deste líquido drenando do teto nasal era diagnóstico da FL. Atenção deve ser dada ao diagnóstico diferencial com rinite, como sugerido por Ramsden (2000), que relatou o caso de uma mulher de 47 anos, sem história de trauma, com rinorréia bilateral, tratada como rinite por anos e que, na verdade, tratava-se de uma FL espontânea bilateral ${ }^{6}$.

A correção da FL pela técnica endonasal já é realizada em larga escala e a literatura mundial mostra que é um método seguro e eficiente ${ }^{4}$. A localização e visualização fornecida pelo endoscópio permitem ao cirurgião uma maior facilidade em remover o tecido fibrótico e escarificar os bordos da lesão permitindo uma maior aderência do enxerto nesta região, tendo desta forma, um excelente resulta$\mathrm{do}^{1,10}$.

Existem várias técnicas endonasais diferentes para correção de FL, e todas tem sido realizadas com grande sucesso $^{2,5}$. Além disso, há uma variedade de enxertos, autólogos e heterólogos, todos sendo formados por tecidos conectivos. Em nossa casuística, assim como na literatura referida, não observamos falha cirúrgica associada com nenhum tipo especifico de enxerto utilizado. Como observado por Schick (2001) e Hao (1996), a exposição incompleta e o largo defeito ósseo foram os fatores preponderantes nos seus insucessos terapêuticos.

Em nossa série, o sucesso durante a primeira intervenção foi de aproximadamente 80\%, semelhante aos resultados de Schick et al. (2001), Burns et al. (1996), Hao et al. (1996) e Ryet et al. (1997). As falhas foram, provavelmente, decorrentes dos grandes defeitos, com herniacão do saco meningoencefálico, localizado na área cribriforme ou no seio esfenóide (Figura 5), ou por causa de fraturas frontobasais extensas. O não-reconhecimento de outro defeito dural, adjacente ao defeito principal, foi a causa do insucesso em um de nossos casos, o que também foi motivo de falha na série de casos de Hao et al. (1997). Nestes casos, devido ao alto débito das fístulas, o posicionamento do enxerto foi prejudicado e a aderência não foi suficiente. Em nossa observação as fístulas pós-operatórias de cirurgia endoscópicas acometeram a lâmina cribriforme e o etmóide posterior, região mais frágil da base do crânio ${ }^{4,10}$ e onde deve haver especial atenção quando a FL for suspeitada.

Meningite é a complicação mais temida para os pacientes que apresentam FL, representando um potencial risco de vida ${ }^{11}$. Estudos demonstram que pode incidir em até $40 \%$ dos pacientes. Tivemos apenas 1 caso de meningite, associado ao largo defeito ósseo, dado que corrobora a pequena morbidade da técnica endonasal. Cuidado especial deve ser dado aos pacientes no pós-operatório, visto que em um dos pacientes a não-observação do posicionamento correto da bolsa coletora de líquor foi a responsável pela síndrome de hiper-drenagem, associada a cefaléia importante.

O uso do antibiótico profilático é controverso ${ }^{11}$. Em todos os pacientes utilizamos antibióticos, porém Nachtigal (1999), que não utilizou profilaxia, não teve nenhum caso de meningite em sua série de 12 pacientes. Choi (1996), em 115 pacientes com FL acidental, observou maior incidência de meningite nos pacientes que utilizaram a profilaxia antibiótica, do que aqueles que não a utilizaram.

\section{CONCLUSÃO}

Concluímos que a elevada taxa de sucesso e a baixa morbidade da correção de fístula por via endonasal faz desta abordagem a técnica de eleição para o tratamento de fístula liquórica rinogênica. FL associada a largos defeitos, meningoceles e lesões secundárias devem ter atenção especial e modificações na técnica, algumas vezes, serão necessárias para garantir o fechamento contínuo da fístula.

\section{REFERÊNCIAS BIBLIOGRÁFICAS}

1. Wax MK, Ramadan HH, Ortiz O, Wetmore SJ. Contemporary management of cerebrospinal fluid rhinorrhea. OtolaryngologyHead and Neck Surgery 1997; 116 (4): 442-9.

2. Schick B, Ibing R, Brors D, Draf W. Long-term study of endonasal duraplasty and review of the literature. Ann Otol Rhinol Laryngol 2001; 110: 142-7.

3. Vrabec DP, Hallberg OE. Cerebrospinal fluid rhinorrhoea by endonasal surgery. Arch Otolaryngol 1964; 80: 218-29.

4. Nachtigal D, Frenkiel S, Mohr G. Endoscopic repair of cerebrospinal fluid rhinorrhea: Is it the treatment of choice. The journal of Otolaryngology 1999; 28 (3): 129-33. 
5. Jones ME, Reino T, Gnoy A et al. Identification of intranasal cerebrospinal leaks by topic application with fluorescein dye. American journal of rhinology 2000; 14 (2): 93-6.

6. Ramsden JD, Corbridge R, Bates G. Bilateral cerebrospinal fluid rhinorrhoea 2000; 114: 137-8.

7. Burns JA, Dodson EE, Gross CW. Transnasal endoscopic repair of cranionasal fístulae: a refined technique with long-term followup. Laryngoscope 1996; 106: 1080-3.

8. Reyt E, Righini C, Colombani JM, Favre JJ. Transnasal endoscopic identification and repair of cerebrospinal rhinorrhoea. Skull Base Surgery 1997; 7 (Suppl 2): 52.
9. Ommaya AK. Spinal fluid fístulae. Clinics of Otolaryngology 1983; 8: 317-27.

10. Hao SP. Transnasal endoscopic repair of cerebrospinal fluid rhinorrhoea: an interposition technique. Laryngoscope 1996; 106: 501-3.

11. Choi D, Span R. Traumatic cerebralspinal fluid leakage: risks factors and the use of prophylactic antibiotics. Br J of Neurosurgery 1996; 10: $571-5$.

Revista Brasileira de Otorrinolaringologia 71 (4) Parte 1 Julho/Agosto 2005

http://www.rborl.org.br / e-mail: revista@aborlccf.org.br 